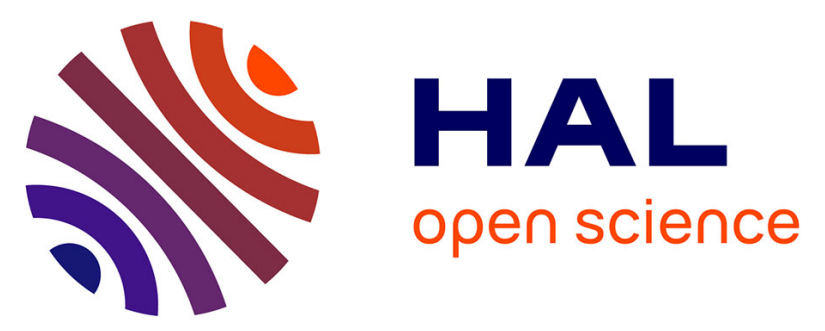

\title{
Thermodiffusion of citrate-coated $\gamma$-Fe 2 O 3 nanoparticles in aqueous dispersions with tuned counter-ions - anisotropy of the Soret coefficient under a magnetic field
}

\author{
M. Kouyaté, C. L Filomeno, G. Demouchy, G. Mériguet, S. Nakamae, V. \\ Peyre, M. Roger, A. Cēbers, J. Depeyrot, E. Dubois, et al.
}

\section{To cite this version:}

M. Kouyaté, C. L Filomeno, G. Demouchy, G. Mériguet, S. Nakamae, et al.. Thermodiffusion of citrate-coated $\gamma$-Fe 2 O 3 nanoparticles in aqueous dispersions with tuned counter-ions - anisotropy of the Soret coefficient under a magnetic field. Physical Chemistry Chemical Physics, 2019, 21 (4), pp.1895-1903. 10.1039/C8CP06858E . hal-02275983

\section{HAL Id: hal-02275983 \\ https://hal.sorbonne-universite.fr/hal-02275983}

Submitted on 2 Sep 2019

HAL is a multi-disciplinary open access archive for the deposit and dissemination of scientific research documents, whether they are published or not. The documents may come from teaching and research institutions in France or abroad, or from public or private research centers.
L'archive ouverte pluridisciplinaire HAL, est destinée au dépôt et à la diffusion de documents scientifiques de niveau recherche, publiés ou non, émanant des établissements d'enseignement et de recherche français ou étrangers, des laboratoires publics ou privés. 


\title{
Thermodiffusion of citrate-coated $\gamma-\mathrm{Fe}_{2} \mathrm{O}_{3}$ nanoparti- cles in aqueous dispersions with tuned counter-ions - Anisotropy of the Soret coefficient under magnetic field
}

\author{
M. Kouyaté,$^{a}$ C. L. Filomeno, ${ }^{a, b}$ G. Demouchy, ${ }^{a}$, G. Mériguet,${ }^{a}$ S. Nakamae,${ }^{c}$ V. Peyre,${ }^{a}$ \\ M. Roger, ${ }^{c}$ A. Cēbers,${ }^{d}$ J. Depeyrot,${ }^{e}$ E. Dubois,${ }^{a}$ and R. Perzynski ${ }^{* a}$
}

\begin{abstract}
Under a temperature gradient, the direction of thermodiffusion of charged $\gamma-\mathrm{Fe}_{2} \mathrm{O}_{3}$ nanoparticles (NPs) depends on the nature of the counter-ions present in the dispersion, resulting in either positive or negative Soret coefficient. Various counter-ions are probed in finely tuned and well characterized dispersions of citrate-coated NPs at comparable concentrations of free ionic species. The Soret coefficient $S_{\mathrm{T}}$ is measured in stationary conditions together with the mass-diffusion coefficient $D_{m}$ using a Forced Rayleigh Scattering method. The strong interparticle repulsion, determined by SAXS, is also attested by the increase of $D_{m}$ with NP's volume fraction $\Phi$. The $\Phi$-dependence of $S_{\mathrm{T}}$ is analyzed in terms of thermophoretic and thermoelectric contributions of the various ionic species. The obtained single-particle thermophoretic contribution of the NPs (the Eastman entropy of transfer $\hat{S}_{N P}$ ) varies linearly with the entropy of transfer of the counterions. This is understood in terms of electrostatic contribution and of hydration of the ionic shell surrounding the NPs. Two aqueous dispersions, respectively with $S_{\mathrm{T}}>0$ and with $S_{\mathrm{T}}<0$ are then probed under an applied field $\vec{H}$, an anisotropy of $D_{m}$ and of $S_{\mathrm{T}}$ is induced while the in-field system remains monophasic. Whatever the $\vec{H}$-direction (parallel or perpendicular to the gradients $\vec{\nabla} T$ and $\vec{\nabla} \Phi)$, the Soret coefficient is modulated keeping the same sign as in zero applied field. Infield experimental determinations are well described using a mean field model of the interparticle magnetic interaction.
\end{abstract}

\section{Introduction}

Magnetic fluids ${ }^{1-4}$ based on polar media have been proposed as an alternative to conventional electrolytes in thermoelectric devices $^{5,6}$. In such dispersions, the nanoparticles (NPs), ca. $10 \mathrm{~nm}$ in diameter, bear a large magnetic moment and they also bear a large surface charge. The response of these dispersions to an applied gradient of temperature $\vec{\nabla} T$ is then twofold, thermoelectric and thermodiffusive ${ }^{7-12}$. The Seebeck effect ${ }^{7,13}$ induces a local electric field $\vec{E}=S_{e} \vec{\nabla} T$ in the dispersion $\left(S_{e}\right.$ being the Seebeck coefficient) and the Ludwig-Soret effect ${ }^{14,15}$ induces a local

\footnotetext{
a Sorbonne Université, CNRS, PHysico-chimie des Electrolytes et Nanosystèmes InterfaciauX, F-75005, Paris, France. Fax: +33 14427 3228; Tel: +33 14427 3267; E-mail: regine.perzynski@upmc.fr

${ }^{b}$ Inst. de Quimica, Complex Fluid Group, Universidade de Brasília, Brasília, Brazil. c SPHINX, CEA-IRAMIS-SPEC, CNRS UMR 3680, CEA Saclay, Gif sur Yvette, France.

${ }^{d}$ Latvia University - Chair of Theoretical Physics - Riga - Latvia.

e Inst. de Fisica, Complex Fluid Group, Universidade de Brasília, Brasília, Brazil. $\ddagger$ Also at Département de Physique - Univ. Cergy-Pontoise - 33 Bd du port 95011 Cergy-Pontoise, France
}

gradient of volume fraction $\vec{\nabla} \Phi=-\Phi S_{\mathrm{T}} \vec{\nabla} T$ ( $S_{\mathrm{T}}$ being the Soret coefficient). These two effects are intricately connected via the Eastman entropies of transfer $\hat{S}_{i}{ }^{*}$ of the charged species $\{i\}$ in the system ${ }^{16-21}$.

It is easy to see that controlling both the magnitude and the direction of thermodiffusion, either towards cold regions $\left(S_{\mathrm{T}}>0\right)$ or towards hot regions $\left(S_{\mathrm{T}}<0\right)$, can be of paramount importance for thermoelectric applications ${ }^{22-25}$. In ${ }^{26,27}$, we have shown that the sign of Soret coefficient is controlled by the nature of the interface between the charged NPs and the solvent in which they are dispersed. It is then related 1) to the nature of the solvent, 2) to that of the NP-coating and 3) to the kind of counter-ions used to stabilize the dispersion. In dispersions of magnetic NPs, an applied magnetic field gives a supplementary external parameter for tuning thermodiffusion ${ }^{28-30}$.

We investigate here in detail a system for which the magnitude

\footnotetext{
$* \hat{S}_{i}$ is defined as in ${ }^{16} Q_{i}^{*} / T$ with $Q_{i}^{*}$ the heat of transport of the ionic species $\{i\}$.
} 
and sign of the Soret coefficient is controlled by the nature of the counter-ions, together with an applied magnetic field $\vec{H}$ capable of modulating the $S_{\mathrm{T}}$ value whatever its sign. The present system is an aqueous dispersion of maghemite $\left(\gamma-\mathrm{Fe}_{2} \mathrm{O}_{3}\right)$ nanoparticles at $\mathrm{pH}=7$ with a volume fraction $\Phi$, here ranging from 0.5 to $6 \%$. Thanks to a citrate-coating, the NPs bear a negative surface charge surrounded by positive counter-ions, that provides the NPs with a dominant electrosteric repulsion, ensuring the colloidal stability of the system even under a large applied magnetic field $^{31-33}$.

Using a forced Rayleigh Scattering method ${ }^{34,35}$, we determine the $\Phi$-dependence of the Soret coefficient $S_{\mathrm{T}}$ and that of the mass diffusion coefficient $D_{m}$, at room temperature, for different monovalent counter-ions $\mathrm{X}^{+}$, with $\mathrm{X}^{+}$being either $\mathrm{Li}^{+}$(lithium), or $\mathrm{Na}^{+}$(sodium), or $\mathrm{TMA}^{+}$(tetramethylammonium) or $\mathrm{TBuA}^{+}$ (tetrabutylammonium). In electrolytes, specific effects of ions are reported, they are numerous and miscellaneous, on viscosity B-coefficients, on the structure of water, ... ${ }^{36-40} . \mathrm{Li}^{+}$and $\mathrm{Na}^{+}$ are chosen because they are hydrophilic cations while $\mathrm{TMA}^{+}$and $\mathrm{TBuA}^{+}$ions are frequently presented as archetypal hydrophobic cations $^{41}$. The synthesis method allows the production of a series of dispersions based on the same NPs, only changing the nature of $\mathrm{X}^{+26}$ and keeping comparable concentration of free ionic species. The interparticle repulsion is checked via Small Angle $\mathrm{X}$-ray Scattering measurements. The NPs electrophoretic charge and its temperature-dependence (in the vicinity of room temperature) are determined via measurements at very low $\Phi$ of the NPs electrophoretic mobility and mass diffusion coefficient ${ }^{42}$.

We discuss the results obtained in zero-field in terms of single nanoparticle thermophoretic contribution (NP's Eastman entropy of transfer $\hat{S}_{\mathrm{NP}}$ ) and of thermoelectric contribution to $S_{\mathrm{T}}$. A particular focus is given on the $\mathrm{X}^{+}$-dependence of $\hat{S}_{\mathrm{NP}}$ and on the various contributions to NP's ionic shielding ${ }^{11,43,44}$.

Finally we present the in-field anisotropy of the diffusion coefficient $D_{m}$ and of the Soret coefficient $S_{\mathrm{T}}$. The field-dependence of $S_{\mathrm{T}}$ is here adjusted with a mean field model ${ }^{45}$, similar to the one previously developed to describe the in-field $D_{m}$-anisotropy ${ }^{46,47}$.

\section{Experimental}

\subsection{Sample preparation and characterizations}

\subsubsection{Chemical synthesis}

The roughly spherical magnetic NPs are obtained by polycondensation of acidic solutions of $\mathrm{FeCl}_{2}$ and $\mathrm{FeCl}_{3}$ in a strongly alkaline aqueous medium following Massart's method ${ }^{48,49}$. The obtained maghemite $\left(\gamma-\mathrm{Fe}_{2} \mathrm{O}_{3}\right)$ nanoparticles are dispersed in an aqueous nitric acid solution, at $1.5 \leq \mathrm{pH} \leq 2$. To modify the NP's solid/liquid interface within the aqueous solvent, we proceed as described in 26,27 . The NPs are citrate-coated at room temperature by adding to the acidic dispersion an excess of solid citric acid $\left(\mathrm{H}_{3} \mathrm{Cit}\right)$, leading to flocculation. Nitrate ions are washed off from the stirred flocculate in several steps. At the end, the addition of a controlled aqueous strong base $\mathrm{XOH}$ at $0.1 \mathrm{~mol} \mathrm{~L}^{-1}$ (here $\mathrm{X}=\mathrm{Li}, \mathrm{Na}$, TMA or TBuA) allows reaching $\mathrm{pH}=7$ and obtaining stable dispersions of negatively charged NPs, with different counter-ions $\mathrm{X}^{+}$, at a typical volume fraction $\Phi \sim 4-6 \%$. The nature and the amount of the $\mathrm{X}^{+}$ions introduced in solution are precisely known. All dispersions presented here are based on the same initial batch of NPs. An ultracentrifugation of a portion of the obtained dispersions $\left(2 \times 10^{5} \mathrm{~g}\right)$ is performed. Providing that the ultracentrifugation does not perturb local equilibria around NPs, conductivity measurements in the supernatant allow determining concentrations of free citrate co-ions and counterions in each dispersions. A solution similar to the supernatant is then used to obtain dilute dispersions at lower $\Phi$ 's. Table 1 collects the concentration $\left[\mathrm{Cit}^{3-}\right]_{\text {free }}$ of free co-ions in the obtained dispersions. The structural surface-charge density is then deduced from the total quantity of counterions introduced. For all the dispersions, it is $-1 \pm 0.4 e \mathrm{~nm}^{-2}$ and imparts the dispersions with a strongly repulsive interaction potential between pairs of NPs. The in-field colloidal stability of the dispersions is probed by in-field light scattering ${ }^{50}$ up to $H=300 \mathrm{kA} \mathrm{m}^{-1}$. The size distribution of the magnetic nanoparticles is determined by magnetization measurements (Vibrating Sample Magnetometer) at room temperature. It is adjusted with a log-normal distribution of diameter, of polydispersity index 0.4 , leading to a volume-averaged diameter $d_{\mathrm{NP}}=\sqrt[3]{\left\langle d_{0}^{3}\right\rangle}=8.5 \mathrm{~nm}^{26}$.

\subsubsection{Characterization of NP's dispersions by Small Angle X- rays Scattering}

The nanoparticle size distribution and the strong interparticle repulsion (which is on average stronger than that of hard spheres ${ }^{27}$ ) are probed by Small Angle X-rays Scattering (SAXS). These experiments are performed at the SOLEIL synchrotron (SWING beam-line, Gif-sur- Yvette, France) at a beam energy $15 \mathrm{keV}$ with a scattering vector $Q$ ranging between $3.2 \times 10^{-3} \AA^{-1}$ and $4 \times 10^{-1} \AA^{-1}$. Thanks to a Zimm-plot, we deduce the form factor of the nanoparticles as a function of the scattering vector $\mathrm{Q}^{26}$. Analyzing the SAXS results at finite volume fraction as formerly described in ${ }^{26,27}$ allows to deduce the osmotic compressibility $\chi$ of the NP system as a function of the NP volume fraction $\Phi$. One can model it (as in ${ }^{26,27,51}$ ) with the CarnahanStarling expression for effective hard spheres ${ }^{52,53}$ of volume fraction $\Phi_{\text {eff }}$ (and effective diameter taking in account the screening length $\kappa^{-1}$ ) as $\chi_{\mathrm{CS}}\left(\Phi_{\text {eff }}\right)=\chi(\Phi)$. It leads to the value of $A_{2}$, the second virial coefficient of the osmotic pressure $\Pi$ in the dispersion:

$$
A_{2}=A_{2}^{H S} \frac{\Phi_{\text {eff }}}{\Phi}=4 \frac{\Phi_{\text {eff }}}{\Phi} \quad \text { with } \quad \Phi_{\text {eff }}=\Phi\left(1+\frac{2 \kappa^{-1}}{d_{\mathrm{NP}}}\right)^{3},
$$

where $A_{2}^{H S}=4$ is the Hard Sphere value of $A_{2}$ and $d_{\mathrm{NP}}$ the NP diameter. The values of $A_{2}$ are also collected in Table 1 .

\subsection{Electrophoretic charge determination}

The dynamic electrophoretic charge number $\xi_{0}$ of the NPs and its derivative $\mathrm{d} \xi_{0} / \mathrm{d} T$ are deduced from measurements of the NP electrophoretic mobility $\mu^{e l}$ and of the mass diffusion coefficient $D_{m}$ in very dilute colloidal dispersions, and for a temperature $T$ ranging from $20^{\circ} \mathrm{C}$ to $40^{\circ} \mathrm{C}$, using:

$$
\xi_{0}=\frac{k T \mu^{e l}}{e D_{m}} \quad \text { at } \Phi \rightarrow 0 .
$$


Table 1 Sample characteristics : $\mathrm{X}^{+}$, NP's counter-ions in the sample; $D_{m, 0}$, NP's diffusion coefficient at infinite dilution limit as obtained using Eq. 3 with $\chi=\chi_{\mathrm{CS}}\left(\Phi_{\mathrm{eff}}\right)$ (see Eq. 5 ); $\left[\mathrm{Cit}^{3-}\right]_{\text {free }}$, concentration of free citrate species in the sample; $A_{2}$, second virial coefficient of the osmotic pressure (related to the 2-body term in the pair interaction potential between the NPs) as obtained from SAXS determinations of $\Phi_{\text {eff }}$ and Eq. $1 ; \kappa^{-1}$, screening length deduced from Eq. $1 ; \xi_{0}$, electrophoretic charge number of the NPs and $d \xi_{0} / d T$, its derivative with respect to temperature, as determined from electrophoretic and diffusion coefficient measurements at very low $\Phi$ 's (see text); $\hat{S}_{+} / k T$ and $\hat{S}_{\mathrm{NP}} / k T$, the Eastman entropy of transfer of the counterions over $k T$ (from ref. ${ }^{16}$ ) and that of NP's as obtained from the adjustments of $S_{T}$ measurements of Fig 1 by Eqs. 11 and 13 ; $\hat{S}_{\mathrm{NP}}^{\mathrm{cm}} / k T$, electrostatic contribution to $\hat{S}_{\mathrm{NP}} / k T$ as obtained from the capacitor model of Eq. 19.

\begin{tabular}{lccccccccc}
\hline \begin{tabular}{c} 
Counter-ions \\
\multicolumn{1}{c}{$\mathrm{X}^{+}$}
\end{tabular} & $\begin{array}{c}D_{m, 0} \\
\left(\times 10^{-11} \mathrm{~m}^{2} \mathrm{~s}^{-1}\right)\end{array}$ & $\begin{array}{c}{\left[\mathrm{Cit}^{3-}\right]_{\text {free }}} \\
\left.(\mathrm{mol} \mathrm{L})^{-1}\right)\end{array}$ & $\mathrm{A}_{2}$ & $\begin{array}{c}\kappa^{-1} \\
(\mathrm{~nm})\end{array}$ & $\begin{array}{c}\xi_{0} \\
\left(\text { at } 22.5{ }^{\circ} \mathrm{C}\right)\end{array}$ & $\begin{array}{c}\mathrm{d} \xi_{0} / \mathrm{dT} \\
\left(\mathrm{K}^{-1}\right)\end{array}$ & $\begin{array}{c}\hat{S}_{+} / k T \\
\left(\mathrm{~K}^{-1}\right)\end{array}$ & $\begin{array}{c}\hat{S}_{\mathrm{NP}} / k T \\
\left(\mathrm{~K}^{-1}\right)\end{array}$ & $\begin{array}{c}\hat{S}_{\mathrm{NP}}^{\mathrm{cm}} / k T \\
\left(\mathrm{~K}^{-1}\right)\end{array}$ \\
\hline $\mathrm{TBuA}^{+}$ & 1.8 & 0.028 & 14 & 2.2 & -40 & +0.27 & +0.028 & +0.09 & -0.42 \\
$\mathrm{TMA}^{+}$ & 1.9 & 0.024 & 10.5 & 2.0 & -32 & -0.03 & +0.014 & -0.02 & +0.14 \\
$\mathrm{Na}^{+}$ & 1.8 & 0.027 & 13 & 1.6 & -30 & -0.68 & +0.005 & -0.13 & +1.20 \\
$\mathrm{Li}^{+}$ & 1.9 & 0.031 & 15.5 & 2.4 & -28 & -0.21 & +0.001 & -0.20 & +0.45 \\
\hline
\end{tabular}

The electrophoretic mobility measurements are performed at $\Phi \sim 0.01 \%$ with a NanoZS from Malvern (with a dip cell) and the mass diffusion coefficient is obtained by Quasi-Elastic Light Scattering (QELS) at $\Phi \sim 0.2 \%$ with a Vasco DLS particle analyzer from Cordouan Technologies dedicated to dark media, as in ${ }^{42}$. The values of $\xi_{0}$ and $\mathrm{d} \xi_{0} / \mathrm{d} T$, obtained for the various counterions $\mathrm{X}^{+}$probed here, are collected in Table 1

\subsection{Forced Rayleigh Scattering experiment}

To measure the diffusion coefficient $D_{m}$ at finite concentration and the Soret coefficient $S_{T}$, we use here the home-made Forced Rayleigh Scattering (FRS) ${ }^{\dagger}$ setup which is extensively described in Ref. ${ }^{34}$.

Light from a high power $\mathrm{Hg}$ arc lamp (here modulated at $100 \mathrm{~Hz}$ ) come through a grid and is focused by a camera lens. It produces a light intensity grating in the NP's dispersion, which is put in a thin optical cell of thickness $t=10-100 \mu \mathrm{m}$. Thanks to the strong optical NP absorption, a thermal grating is quickly imprinted in the aqueous sample (in a few $100 \mu$ s) with a spatial period $\Lambda$ ranging between 80 and $180 \mu \mathrm{m}$. Because of the Soret effect, the nanoparticles then tend to migrate either towards the hot (thermophilic behavior) or the cold regions (thermophobic behavior) depending on the colloidal characteristics of the system. This results in a spatial modulation of NP concentration in the sample. The first order diffraction of a (non-absorbed) He-Ne laser beam probes both temperature and concentration gratings. Their temporal evolution timescales differ by more than an order of magnitude and they can be decoupled. It is then possible to determine the Soret coefficient $S_{\mathrm{T}}{ }^{34}$ in stationary conditions, providing a precise knowledge of the dependence of the optical index of the dispersion on both temperature and NP concentration. Measurements are performed at different $\Lambda$ 's and different powers of the $\mathrm{Hg}$ arc-lamp, in order to check the measured $S_{T}$ coefficient does not depend on these two parameters. A magnetic field $\vec{H}$ can be applied in the same plane as the temperature and concentration gratings either parallel or perpendicular to the scattering vector $\vec{Q}$ (direction perpendicular to the fringes and

$\dagger$ FRS implies forced inhomogeneities with respect to the spontaneous fluctuations observed in a classical Rayleigh scattering experiment ${ }^{35}$ modulus $2 \pi / \Lambda)$. The maximum value of $H$ is $72 \mathrm{kA} \mathrm{m}^{-1}$. Infield measurements are performed at a ratio $t / \Lambda<0.6$ in order to be in the $2 \mathrm{D}$-array conditions. Moreover the magnetic thermal Rayleigh number $R_{S, m}$ being here always smaller than $10^{-5.2}$ in our range of parameters $\left(\delta \Phi / \Phi \leq 5 \times 10^{-3}, \delta T / T \leq 8 \times 10^{-5}\right.$ in the concentration and temperature arrays), the measurements are all performed below the threshold of 2D magnetoconvection ${ }^{54,55}$.

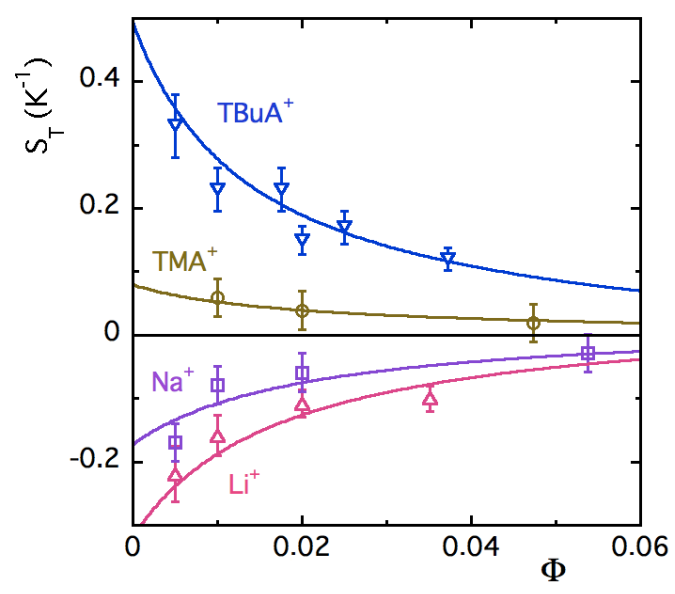

Fig. $1 \Phi$-dependence of Soret coefficient $S_{\mathrm{T}}$ for four counter-ions $\mathrm{X}^{+}: \mathrm{Li}^{+}$ (lithium), $\mathrm{Na}^{+}$(sodium), $\mathrm{TMA}^{+}$(tetramethylammonium), $\mathrm{TBuA}^{+}$(tetrabutylammonium). Full lines correspond to fits of $S_{T}(\Phi)$ using Eqs. 11 and 13 , with $\hat{S}_{\mathrm{NP}} / k T$, as the only free parameter. The obtained values of $\hat{S}_{\mathrm{NP}} / k T$ are given in Table 1 .

\section{Results in zero applied field}

\section{1 $\Phi$-dependence of $S_{\mathrm{T}}$ and $D_{m}$ for the various $\mathrm{X}^{+}$}

The coefficients $S_{\mathrm{T}}$ and $D_{m}$ are determined as a function of the NP's volume fraction $\Phi$ for the various counter-ions $\mathrm{X}^{+}$(Table 1) with $\left[\mathrm{Cit}^{3-}\right]_{\text {free }} \sim 3 \times 10^{-2} \mathrm{~mol} \mathrm{~L}^{-1}$. The $\Phi$-dependence of $S_{\mathrm{T}}$ is presented in Fig. 1 for the 4 different ions $\mathrm{X}^{+}$. The sign of $S_{\mathrm{T}}$ is either positive or negative depending on the nature of $\mathrm{X}^{+}$and the volume fraction is not able to modify its sign, at least in the explored range of $\Phi$.

The same general behaviour of $D_{m}$ as a function of $\Phi$ is ob- 
tained whatever $\mathrm{X}^{+}$; Namely, $D_{m}$ is an increasing function of $\Phi$ from an initial value $D_{m, 0}$ at $\Phi=0$. Fig. 2 illustrates this point for $\mathrm{X}^{+}=\mathrm{TBuA}^{+}, \mathrm{Na}^{+}$and $\mathrm{Li}^{+}$(which have very close $\left[\mathrm{Cit}^{3-}\right]_{\text {free }}$ values - see Table 1). This behaviour confirms the strong interparticle repulsion inside the dispersions. Indeed the diffusion coefficient $D_{m}$ is related to the osmotic pressure $\Pi$ and the local friction $\zeta$ of the NPs in the colloidal dispersion through the following relation :

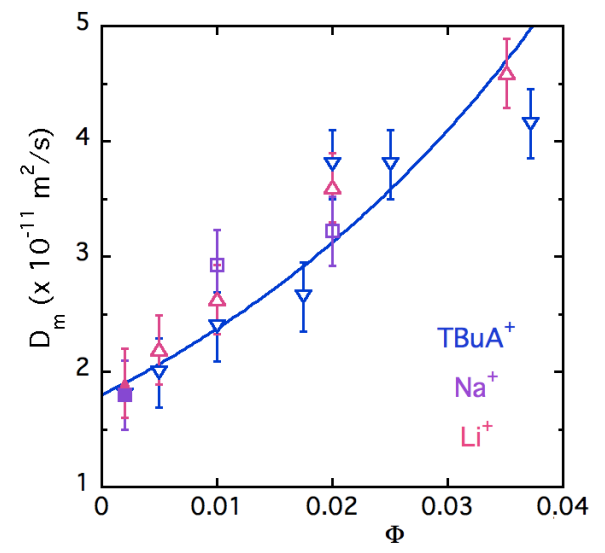

Fig. $2 \Phi$-dependence of mass-diffusion coefficient $D_{m}$ for $\mathrm{X}^{+}=\mathrm{TBuA}^{+}$, $\mathrm{Na}^{+}$and $\mathrm{Li}^{+}$; Open symbols: FRS measurements (same as in Fig. 1); Full symbols: QELS measurements. The fit corresponds to Eq. 3 with Eq. 5, Eq. 1 and Table 1 for $\mathrm{X}^{+}=\mathrm{TBuA}^{+}$.

$$
D_{m}=\frac{1}{\zeta} \frac{\partial \Pi}{\partial n}=\frac{1}{\zeta} \frac{k T}{\chi(\Phi)} .
$$

where $n=\Phi / V_{\mathrm{NP}}$ is the number of NPs per unit volume ( $V_{\mathrm{NP}}$ being the NP's volume) and the osmotic compressibility $\chi$ of NPs system is related to $\Pi$, thanks to :

$$
\chi(\Phi)=\frac{k T}{\frac{\partial \Pi}{\partial n}} .
$$

In the framework of Carnahan-Starling formalism of effective hard spheres, it writes :

$$
\chi_{\mathrm{CS}}\left(\Phi_{\mathrm{eff}}\right)=\frac{\left(1-\Phi_{\mathrm{eff}}\right)^{4}}{1+4 \Phi_{\mathrm{eff}}+4 \Phi_{\mathrm{eff}}^{2}-4 \Phi_{\mathrm{eff}}^{3}+\Phi_{\mathrm{eff}}^{4}} .
$$

The full line in Fig. 2 corresponds to $D_{m}=k T /\left[\zeta \chi_{\mathrm{CS}}\left(\Phi_{\text {eff }}\right)\right]$ for $\mathrm{X}^{+}=\mathrm{TBuA}^{+}$with $\zeta=6 \pi \eta_{0} R_{\mathrm{H}}\left(R_{\mathrm{H}}\right.$ being the NP's hydrodynamic radius, $\eta_{0}$ the water viscosity; the $\Phi$-dependence of the friction $\zeta$ has been here neglected). At $\Phi=0, \chi$ equals 1 and $D_{m, 0}$ then writes as $D_{m, 0}=k T / 6 \pi \eta_{0} R_{\mathrm{H}}$. Table 1 collects the $D_{m, 0}$ values for the different counter-ions $\mathrm{X}^{+}$, showing that the values are similar within the error bar $\pm 0.2 \times 10^{-12} \mathrm{~m}^{2} / \mathrm{s}$.

\section{2 $S_{\mathrm{T}}$ - adjustments}

In stationary conditions $8,9,42,56,57$, the ionic NPs being submitted to a small concentration gradient $\vec{\nabla} n$ and a small tempera- ture gradient $\vec{\nabla} T$, the cooperative flux $\vec{j}_{N P}$ of nanoparticles can be written as ${ }^{57-59}$ :

$$
\vec{j}_{N P}=-\frac{1}{\zeta}\left(\vec{\nabla} \Pi+n \hat{S}_{\mathrm{NP}} \vec{\nabla} T-n e \xi_{0} S_{e}^{s t} \vec{\nabla} T\right)=\overrightarrow{0}
$$

where $\hat{S}_{\mathrm{NP}}$ is the Eastman entropy of transfer of the NPs, $e$ is the electron charge in absolute value, $e \xi_{0}$ the electrophoretic effective charge of the NPs and $S_{e}^{s t}$ the Seebeck (thermoelectric) coefficient ${ }^{7}$.
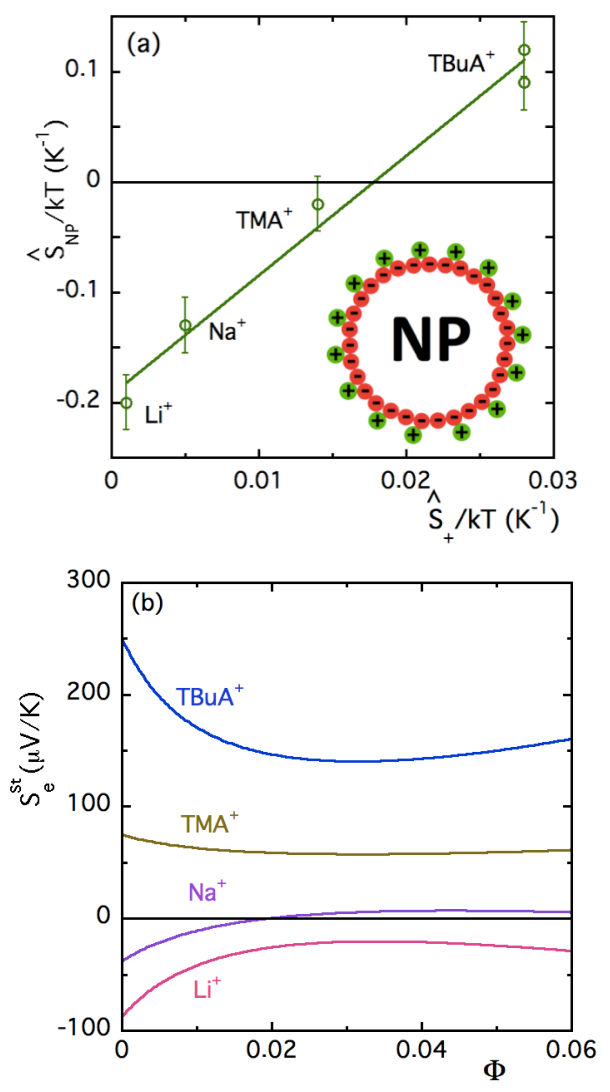

Fig. 3 (a) $\hat{S}_{+}$-dependence of $\hat{S}_{\mathrm{NP}} / k T$ for the various samples; Inset: sketch of the NP's coated with negative citrate species and then "dressed" with condensed counter ions, leading an effective charge (smaller than the structural one due to citrate); (b) $\Phi$-dependence of $S_{e}^{S t}$ coefficient for the various samples as obtained from Eq. 13 using adjusted values of $\hat{S}_{\mathrm{NP}} / k T$ from Table 1 .

The system being stationary, fluxes of all species equal zero ${ }^{9}$; NPs flux $\vec{j}_{N P}$, together with flux $\vec{j}_{+}$of the counterions (valence $z_{+}$and number per unit volume $n_{+}$) and flux $\vec{j}_{-}$of the coions (valence $z_{-}$and number per unit volume $n_{-}$). Writing $\vec{\nabla} \Pi=\frac{\partial \Pi}{\partial n} \vec{\nabla} n+\frac{\partial \Pi}{\partial T} \vec{\nabla} T$, it becomes ${ }^{\ddagger}$ :

$$
\vec{j}_{N P}=-D_{m}\left[\vec{\nabla} n+\frac{n \chi}{k T}\left(\frac{1}{n} \frac{\partial \Pi}{\partial T}+\hat{S}_{\mathrm{NP}}-e \xi_{0} S_{e}^{s t}\right) \vec{\nabla} T\right]=\overrightarrow{0}
$$

$\ddagger$ In $\vec{j}_{+}$and $\vec{j}_{-}$, the contribution from the osmotic pressure of the free ions is here negligible. 


$$
\begin{aligned}
& \vec{j}_{+}=-D_{+}\left[\vec{\nabla} n_{+}+\frac{n_{+}}{k T}\left(\hat{S}_{+}-z_{+} e S_{e}^{s t}\right) \vec{\nabla} T\right]=\overrightarrow{0} \\
& \vec{j}_{-}=-D_{-}\left[\vec{\nabla} n_{-}+\frac{n_{-}}{k T}\left(\hat{S}_{-}-z_{-} e S_{e}^{s t}\right) \vec{\nabla} T\right]=\overrightarrow{0}
\end{aligned}
$$

Here $D_{+}$and $D_{-}$are the diffusion coefficients of counter ions and co-ions, $\hat{S}_{+}$and $\hat{S}_{-}$their Eastman entropy of transfer. The Ludwig-Soret coefficient $S_{\mathrm{T}}$, which is defined by :

$$
\vec{\nabla} n=-n S_{\mathrm{T}} \vec{\nabla} T
$$

is deduced from Eq. 7 as :

$$
S_{T}=\chi\left[\frac{1}{n k T} \frac{\partial \Pi}{\partial T}+\frac{\hat{S}_{\mathrm{NP}}}{k T}-e \xi_{0} \frac{S_{e}^{s t}}{k T}\right] .
$$

If the (static) NP's effective charge is $Z$, the neutrality of the dispersion imposes :

$$
n Z+n_{+} z_{+}+n_{-} z_{-}=0 .
$$

Eliminating with this condition, all the gradients of concentration with an adapted linear combination of Eqs. 7, 8 and 9, we obtain the Seebeck coefficient $S_{e}^{s t} 27$.

The Eastman entropy of transfer of trivalent citrate ions are unknown. We thus assimilate citrate ions to three (independent) monovalent ions $\mathrm{CH}_{3} \mathrm{COO}^{-}$at the concentration $n_{-}=3 n_{\mathrm{Cit}^{3-}}$. The Seebeck coefficient ${ }^{9}$ then reduces to the following monovalent expression (with $z_{+}=z_{-}=1$ ):

$$
e S_{e}^{s t}=\frac{n_{+} \hat{S}_{+}-n_{-} \hat{S}_{-}+Z n \chi\left(\frac{1}{n} \frac{\partial \Pi}{\partial T}+\hat{S}_{\mathrm{NP}}\right)}{n_{+}+n_{-}+Z n \chi \xi_{0}}
$$

with Eastman entropy of transfer of $\mathrm{CH}_{3} \mathrm{COO}^{-}$ions, $\hat{S}_{-} / k T=+0.008 \mathrm{~K}^{-117}$.

Numerical simulations ${ }^{19,20}$ show that the Eastman entropy of transfer of ions in pure alkali halide aqueous solutions depends on the ionic concentration. This concentration dependence can be neglected due to the small values of the present $n_{ \pm}(\sim 9 \times$ $10^{-2} \mathrm{~mol} \mathrm{~L}^{-1}$ ), and we then use the values of $\hat{S}_{ \pm} / k T$ at $298 \mathrm{~K}$ and zero ionic concentration as given by Ref. ${ }^{16}$.

Eq. 11 is used to fit $S_{\mathrm{T}}(\Phi)$ in Fig. 1. In this fit, the interparticle interaction is taken into account by using $\chi(\Phi)=\chi_{\mathrm{CS}}\left(\Phi_{\text {eff }}\right)$. The first term in brackets in Eq. $11, \frac{1}{n k T} \frac{\partial \Pi}{\partial T}$ is positive and frequently neglected $^{6}$, however it can be calculated from Carnahan-Starling expression of $\Pi$ for effective hard spheres ${ }^{27}$. It is of the order of $6 \times 10^{-3} \mathrm{~K}^{-1}$ for present samples. The effective NP's charges $Z$ (static) and $\xi_{0}$ (dynamic) are of the same order of magnitude. For sake of simplicity, we assimilate them to each other in the fit of $S_{\mathrm{T}}(\Phi)$ and the only unknown quantity remaining is then $\hat{S}_{\mathrm{NP}}$, the Eastman entropy of transfer of the NPs $\S$. The value of $\hat{S}_{\mathrm{NP}} / k T$ obtained from the fit of $S_{\mathrm{T}}(\Phi)$ is given in Table 1 for each of the four counterions $\mathrm{X}^{+}$. It linearly depends on $\hat{S}_{+} / k T$, as shown by the plot of Fig. 3-a. The NP's Eastman entropy of transfer then intimately depend on the nature of the counterions (i.e. $\hat{S}_{+}$) which are condensed in the ionic layer surrounding the

$\S$ All the other quantities entering in $S_{e}^{s t}$ (Eq. 13) are known. nanoparticles.

Fig. 3-b plots the $\Phi$-dependence of the Seebeck coefficient $S_{e}^{S t}$, for each of the four counterions $\mathrm{X}^{+}$. Its sign at $\Phi=0$ is determined by the sign of $\left(\hat{S}_{+}-\hat{S}_{-}\right)$. Whatever the initial sign of $S_{\mathrm{e}}^{S t}$, the presence of nanoparticles, up to $\Phi \sim 2 \%$, reduces the absolute value of $S_{\mathrm{e}}^{S t}$. Note that $S_{\mathrm{e}}^{S t}$ changes its sign at $\Phi>2 \%$ for $\mathrm{Na}^{+}$, remaining very small above $\Phi \sim 2 \%$ in the experimental range.

For $\mathrm{TBuA}^{+}$counterions, both $\hat{S}_{\mathrm{NP}}$ and $S_{e}^{S t}$ are positive, leading to $S_{\mathrm{T}}>0$ on the whole range of $\Phi$. For $\mathrm{Li}^{+}$counterions, both $\hat{S}_{\mathrm{NP}}$ and $S_{e}^{S t}$ are negative, leading to $S_{\mathrm{T}}<0$ on the whole range of $\Phi$. $S_{e}^{S t}$ being very small for $\mathrm{Na}^{+}$at $\Phi>2 \%$, the same conclusions as for $\mathrm{Li}^{+}$can be drawn. However, for $\mathrm{TMA}^{+}$, the sign of $S_{\mathrm{T}}$ results from a more subtle competition of sign between $\hat{S}_{\mathrm{NP}}$ and $S_{e}^{S t}$, both contributions to $S_{\mathrm{T}}$ being of opposite sign.

\section{In-field results}

An external magnetic field $\vec{H}$ is now applied to the (in-field stable ${ }^{50}$ ) sample during the FRS measurement, leading to fielddependent values of $D_{m}$ and of $S_{\mathrm{T}}$, as in non-ionic magnetic fluids ${ }^{28-30} . D_{m}$ and $S_{\mathrm{T}}$ both depend on the amplitude of the applied field and on its direction. We limit ourselves here to fields applied in-plane with respect to the sample, either parallel \| (field denoted $\vec{H}_{\| \mid}$) or perpendicular $\perp$ (field denoted $\vec{H}_{\perp}$ ) to the temperature- and concentration-gradients. Fig. 4 and 5 show typical in-field anisotropies of $D_{m}$ and of $S_{\mathrm{T}}$ observed for $\vec{H}_{\|}$and for $\overrightarrow{H_{\perp}}$ for two different samples with citrate-coated NPs dispersed at the same ionic strength $\left(\left[\mathrm{Cit}^{3-}\right]_{\text {free }}=0.03 \mathrm{~mol} \mathrm{~L}^{-1}\right)$.

The in-field anisotropy of $D_{m}$ for such ionic samples, measured in the absence of temperature gradient, has been extensively described in $46,47,60$ in a mean-field framework. The effective Langevin parameter $x_{e}$ in the ferrofluid being defined as :

$$
x_{e}=x+\lambda \gamma L\left(x_{e}\right)
$$

with $x=\mu_{0} \mu_{\mathrm{NP}} H / k T$ the Langevin parameter, $\lambda$ the mean-field parameter II, $L(x)=\operatorname{cotan}(x)-1 / x$ the Langevin function and $\gamma=\mu_{0} m_{\mathrm{S}} \mu_{\mathrm{NP}} \Phi / k T$ the dipolar parameter, $\mu_{\mathrm{NP}}$ and $m_{\mathrm{S}}$ being respectively the magnetic moment and the NP's saturation magnetization, the in-field diffusion coefficient $D_{m}$ then writes with the applied field $\vec{H}$ either perpendicular $\left(\vec{H}_{\perp}\right)$ or parallel $\left(\vec{H}_{\| \mid}\right)$to the $\Phi$-gradient :

$$
D_{m}^{\vec{H}_{\perp}}=\frac{k T}{\zeta}\left(\frac{1}{\chi}-\alpha_{\lambda}\right) \quad \text { and } \quad D_{m}^{\vec{H}_{\| 1}}=\frac{k T}{\zeta}\left(\frac{1}{\chi}+\beta_{\lambda}-\alpha_{\lambda}\right)
$$

with the parameters $\alpha_{\lambda}$ and $\beta_{\lambda}$ given by :

$$
\alpha_{\lambda}=\frac{\lambda \gamma L^{2}\left(x_{e}\right)}{1-\lambda \gamma L^{\prime}\left(x_{e}\right)}
$$

and

$$
\beta_{\lambda}=\frac{\gamma L^{2}\left(x_{e}\right)}{\left[1-\lambda \gamma L^{\prime}\left(x_{e}\right)\right]\left[1+(1-\lambda) \gamma L^{\prime}\left(x_{e}\right)\right]} .
$$

II We use $\lambda=0.22$ as in ${ }^{32,46,47}$, value confirmed by the numerical simulations of Ref. ${ }^{60}$ Up to $\gamma \sim 5$, it has been shown in Ref. ${ }^{61}$ that this simple mean-field model gives same initial magnetic susceptibility as the second order perturbation model of Ref. ${ }^{62}$ with $\lambda=1 / 3$ and as the mean-spherical model of Ref. ${ }^{63}$. 

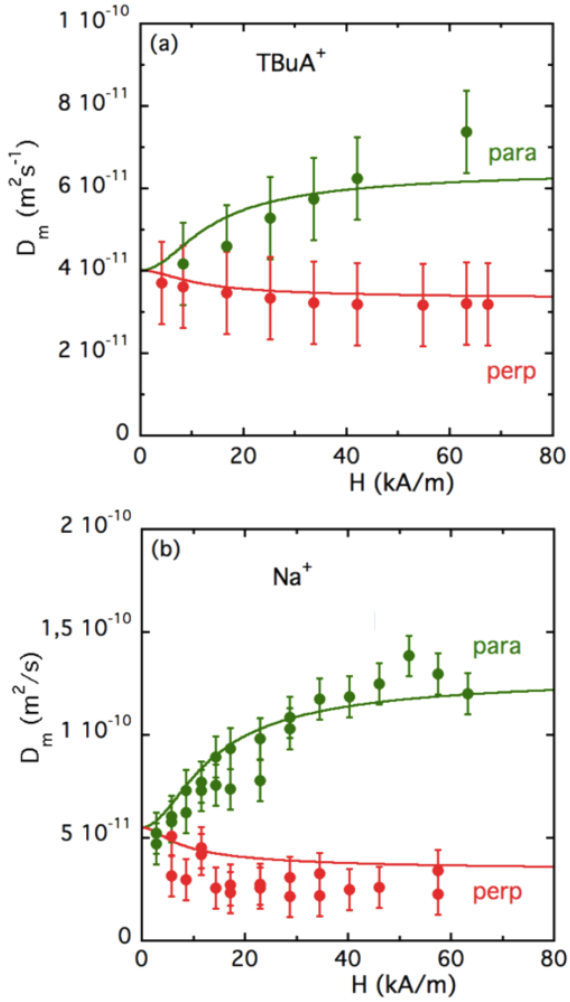

Fig. $4 H$-dependence of $D_{m}$ in the two directions $\|$ and $\perp$, for two different samples with citrate-coated NPs and $\left[\mathrm{Cit}^{3-}\right]_{\text {free }}=0.03 \mathrm{~mol} \mathrm{~L}^{-1}$; (a) sample with same NPs as in Table 1 with $\mathrm{X}^{+}=\mathrm{TBuA}^{+}$at $\Phi=2.5 \%$ with $S_{\mathrm{T}}>0$ and $D_{m}(H=0)=4 \times 10^{-11} \mathrm{~m}^{2} \mathrm{~s}^{-1}$; (b) sample $\mathrm{A}_{5}$ of reference ${ }^{42}$ with $\mathrm{X}^{+}$ $=\mathrm{Na}^{+}$at $\Phi=5.9 \%$ with $S_{\mathrm{T}}<0$ and $D_{m}(H=0)=5.5 \times 10^{-11} \mathrm{~m}^{2} \mathrm{~s}^{-1}$; Full lines correspond to the adjustments by Eqs. 15, 16 and 17, see text for the values of parameters $\lambda$ and $\gamma$.

$\alpha_{\lambda}$ is associated with the mean-field dipolar interaction, which is attractive on average. The term $\beta_{\lambda}$ is anisotropic and due to the discontinuity of $\vec{H}_{\|}$in Maxwell equations along the spatial inhomogeneities of concentration ${ }^{3,45-47}$. $\beta_{\lambda}$ is maximum in the direction parallel to $\vec{\nabla} \Phi$ and null perpendicularly.

Fig. 4-a presents the in-field anisotropy of $D_{m}$ with respect to the field-direction and field-amplitude for a sample with the NPs of Table 1 and $\mathrm{TBuA}^{+}$counter-ions at a NP volume fraction $\Phi=2.5 \%$. It is associated to a positive Soret coefficient $S_{\mathrm{T}}$ and also to a positive $\hat{S}_{\mathrm{NP}}$. Fig. 4-b presents the results for sample $\mathrm{A}_{5}$ of reference ${ }^{42}$ with $\mathrm{Na}^{+}$counter-ions (and slightly larger NPs), at a NP volume fraction $\Phi=5.9 \%$. This sample is associated to a negative Soret coefficient $S_{\mathrm{T}}$ and also to a negative $\hat{S}_{\mathrm{NP}}$. In both cases, the shape of the anisotropy of $D_{m}$ is similar (despite the different nature of the counter-ions) with $D_{m}\left(H_{\perp}\right)<D_{m}(H=0)<D_{m}\left(H_{\|}\right)$ and well adjusted with the model of Eqs. 15, 16 and 17 and a dipolar interaction parameter $\gamma=1.6$ for the sample with $\mathrm{TBuA}^{+}$ counter-ions and 4.2 for the sample with $\mathrm{Na}^{+}$counter-ions $(\chi$ and $\zeta$ keeping their zero field value as in Refs. $\left.{ }^{32,46,47}\right)$. The large difference in $\gamma$ parameter mainly comes from the different $\Phi$-values of the two samples.

The in-field anisotropy of $S_{\mathrm{T}}$ with respect to the field-direction and field-amplitude is presented in Fig. 5 for the same samples as in Fig. 4 (same $\Phi$ 's and $\left[\mathrm{Cit}^{3-}\right]_{\text {free}}$ ). Fig. 5-a presents the results for the sample with $\mathrm{TBuA}^{+}$counter-ions of Fig. 4-a with $S_{\mathrm{T}}(H=$ $0)=+0.16 \mathrm{~K}^{-1}$ and Fig. 5-b the results for the sample with $\mathrm{Na}^{+}$counter-ions of Fig. 4-b with $S_{\mathrm{T}}(H=0)=-2.5 \times 10^{-2} \mathrm{~K}^{-1}$. Whatever the sign of $S_{\mathrm{T}}(H=0)$ and whatever $H$, the value of $\left|S_{\mathrm{T}}\left(H_{\|}\right)\right|$is observed smaller than $\left|S_{\mathrm{T}}(H=0)\right|$ and $\left|S_{\mathrm{T}}\left(H_{\perp}\right)\right|$ larger than $\left|S_{\mathrm{T}}(H=0)\right|$. No sign change of $S_{\mathrm{T}}$ is observed in the experimental range of $\vec{H}$.
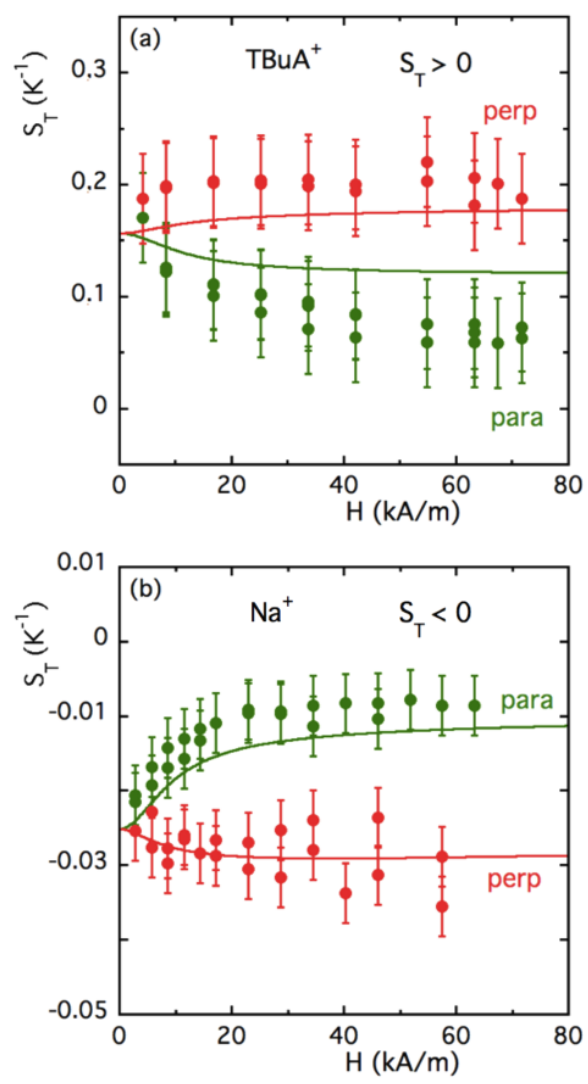

Fig. $5 \mathrm{H}$-dependence of $S_{\mathrm{T}}$ for the two samples of Fig. 4 at $\left[\mathrm{Cit}^{3-}\right]_{\text {free }}=$ $0.03 \mathrm{~mol} \mathrm{~L}^{-1}$; (a) sample with same NPs as in Table 1 with $\mathrm{X}^{+}=\mathrm{TBuA}^{+}$ at $\Phi=2.5 \%$ with $S_{\mathrm{T}}(H=0)=+1.6 \times 10^{-1} \mathrm{~K}^{-1}$; (b) sample $A_{5}$ of reference ${ }^{42}$ with $\mathrm{X}^{+}=\mathrm{Na}^{+}$at $\Phi=5.9 \%$ with $S_{\mathrm{T}}=-2.5 \times 10^{-2} \mathrm{~K}^{-1}$; Full lines correspond to the adjustments by Eq. Eqs. 20, 21, 22 and 23, using the same parameter values as in Fig. 4 .

\section{Discussion}

\subsection{Zero-field results}

The Eastman entropy of transfer of ionic NPs $\hat{S}_{\mathrm{NP}}$, which corresponds to the single-particle thermophoretic contribution, has been shown to depend on all details of the NP-solvent interface $26,27,42$, namely the NP's coating, the nature of the solvent, and in particular here the kind of counter-ions condensed on the NP's. For such charged NPs, several mechanisms of ionic shielding $9,12,44,64,65$ contribute to $\hat{S}_{\mathrm{NP}}$, which can be written as :

$$
\hat{S}_{\mathrm{NP}}=\hat{S}_{\mathrm{NP}}^{\mathrm{cm}}+\hat{S}_{\mathrm{NP}}^{\mathrm{hyd}}
$$


Table $2 \hat{S}_{\mathrm{NP}}^{\text {hyd }} / k T$, the hydration contribution of the NP and of its ionic shell to $\hat{S}_{\mathrm{NP}} / k T$ as obtained from Eqs. 18,19 and Table $1 ; \Delta_{\text {hyd }} S^{o}$ the standard entropy of hydration of ions (data from ${ }^{37,68}$ ).

\begin{tabular}{lcc}
\hline ion & $\hat{S}_{\mathrm{NP}}^{\text {hyd }} / k T\left(\mathrm{~K}^{-1}\right)$ & $\Delta_{\mathrm{hyd}} S^{o}\left(\mathrm{~J} \mathrm{~K}^{-1} \mathrm{~mol}^{-1}\right)$ \\
\hline $\mathrm{TBuA}^{+}$ & +0.51 & -418 \\
$\mathrm{TMA}^{+}$ & -0.16 & -144 \\
$\mathrm{Na}^{+}$ & -1.33 & -111 \\
$\mathrm{Li}^{+}$ & -0.65 & -142 \\
\hline
\end{tabular}

where $\hat{S}_{\mathrm{NP}}^{\mathrm{cm}}$ is the electrostatic contribution, driven by the buildup of the NP's charge and of the ionic shell, usually described with the so-called capacitor model, ${ }^{43,66,67}$ and $\hat{S}_{\mathrm{NP}}^{\text {hyd }}$ the hydration contribution of the NP and of its ionic shell, usually found negative in water ${ }^{66} . \hat{S}_{\mathrm{NP}}^{\text {hyd }}$ can be separated into a volume and a surface contributions ${ }^{42}$.

The capacitor contribution expresses as :

$$
\hat{S}_{\mathrm{NP}}^{\mathrm{cm}}=\frac{\partial}{\partial T}\left[\frac{(e Z)^{2}}{2 \pi \varepsilon_{0} \varepsilon(T) d_{\mathrm{NP}}\left(2+\kappa d_{\mathrm{NP}}\right)}\right]
$$

where $\varepsilon(T)$ is the relative permittivity of water and where $Z$ is the static effective charge (taken equal to $\xi_{0}$ given in Table 1 ). As shown by Table $1, \xi_{0}$ depends here both on temperature and on the nature of the counter-ions. The $\hat{S}_{\mathrm{NP}}^{\mathrm{cm}} / k T$-values range here from -0.45 to $+1.2 \mathrm{~K}^{-1}$ and they strongly depend on $\mathrm{d} \xi_{0} / \mathrm{dT}$.

The hydration contribution to $\hat{S}_{\mathrm{NP}}$, deduced from $\hat{S}_{\mathrm{NP}}^{\text {hyd }}=\hat{S}_{\mathrm{NP}}-$ $\hat{S}_{\mathrm{NP}}^{\mathrm{cm}}$, is presented in Table 2 . It is of the same order of magnitude as $\hat{S}_{\mathrm{NP}}^{\mathrm{cm}}$ but of opposite sign, both being much larger than $\hat{S}_{\mathrm{NP}}$.

Following a similar line as in Ref. ${ }^{17}$, we tentatively compare $\hat{S}_{\mathrm{NP}}^{\text {hyd }} / k T$ with the standard entropy of hydration of free ions $\left(\mathrm{X}^{+}\right)$ as obtained from ${ }^{37,68}$ and given in Table 2. Fig. 6 shows that $\hat{S}_{\mathrm{NP}}^{\text {hyd }} / k T$ is an increasing function of $-T \Delta_{\text {hyd }} S^{o}$ for the counterions of Table 1 (full symbols). The screening length $\kappa^{-1}$ being here of the order of $2 \mathrm{~nm}$ whatever the sample (see inset of Fig. 6 and Table 1), the values previously obtained for $\hat{S}_{\mathrm{NP}}^{\text {hyd }}$ in Ref. ${ }^{42}$ for the A samples with $\mathrm{Na}^{+}$counter-ions at $\kappa^{-1}=1.3 \mathrm{~nm}$ and 3.2 $\mathrm{nm} \|$ are also given for comparison in Fig. 6 (open symbols).

As expected $\hat{S}_{\mathrm{NP}}^{\text {hyd }} / k T$ is negative for $\mathrm{Li}^{+}, \mathrm{Na}^{+}$and $\mathrm{TMA}^{+}$(for which it is close to zero) and the value for $\mathrm{Na}^{+}$is well comparable to those obtained at slightly different $\kappa^{-1}$ in Ref. ${ }^{42}$. However the positive hydration contribution obtained with $\mathrm{TBuA}^{+}$counterions strongly differs. Indeed it has been shown in Ref. ${ }^{41,69}$ that $\mathrm{TBuA}^{+}$ions are large and penetrable (soft) thanks to their four linear arms, and much larger than $\mathrm{TMA}^{+}$ions which are spacefilling (hard), a shape which is closer to that of $\mathrm{Na}^{+}$or $\mathrm{Li}^{+}$ions even if significantly larger.

The present result has also to be compared with the FRS measurements of Ref. ${ }^{21}$ on pure electrolytes, showing that the slope $\partial S_{\mathrm{T}} / \partial T$ of $\left(\mathrm{TBuA}^{+}, \mathrm{OH}^{-}\right)$aqueous solutions is negative, while it is positive for $\left(\mathrm{TMA}^{+}, \mathrm{OH}^{-}\right)$solutions, as usually observed for alkali halide salt solutions under thermal gradients ${ }^{18}$. $\mathrm{TBuA}^{+}$ counter-ions thus appears much more hydrophobic than $\mathrm{TMA}^{+}$

\| i.e. Aqueous dispersions of citrate-coated NPs with a slightly larger $d_{\mathrm{NP}}$.

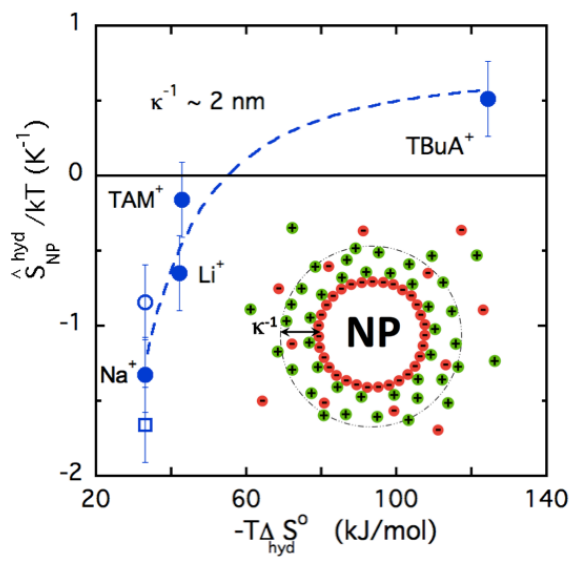

Fig. 6 Main: Hydration contribution $\hat{S}_{\mathrm{NP}}^{\text {hyd }}$ to the NP entropy of transfer $\hat{S}_{\mathrm{NP}}$, normalized by $k T$ as a function of $-T \Delta_{\mathrm{hyd}} S^{o}\left(\Delta_{\mathrm{hyd}} S^{o}\right.$, standard entropy of hydration of free ions $\left.\mathrm{X}^{+}\right)$; Comparison between present results (full circles at $\kappa^{-1}=2 \pm 0.5 \mathrm{~nm}$ ) and those of Ref. ${ }^{42}$ with $\mathrm{Na}^{+}$counterions (open circle at $\kappa^{-1}=1.3 \mathrm{~nm}$ and open square at $\kappa^{-1}=3.2 \mathrm{~nm}$ ); dashed line is a guide for the eye; Inset: Scheme of a citrate-coated NP dressed with its condensed counter-ions and of its surrounding ionic shell, of thickness $\kappa^{-1}$ in the ionic dispersion.

counter-ions $^{70}$, in good agreement with our observations.

\subsection{In-field results}

The in-field anisotropy of $S_{\mathrm{T}}$ has been described recently in the same framework as that of Eq. 15 for $D_{m}(\vec{H})$ in Ref. ${ }^{45}$, including derivatives of the NP's chemical potential with respect to temperature. Note that here the Eastman entropy of transfer of the NP's is supposed independent on $\vec{H}$. The anisotropic in-field Soret coefficient $S_{\mathrm{T}}(\vec{H})$ then writes as :

$$
S_{T}^{\vec{H}_{\perp}}=\frac{1}{k T\left(\frac{1}{\chi}-\alpha_{\lambda}\right)}\left[\frac{1}{n} \frac{\partial \Pi_{H=0}}{\partial T}+\hat{S}_{\mathrm{NP}}-e \xi_{0} S_{e}^{s t}+k S_{1}\right]
$$

and

$$
S_{T}^{\vec{H}_{\| \mid}}=\frac{1}{k T\left(\frac{1}{\chi}+\beta_{\lambda}-\alpha_{\lambda}\right)}\left[\frac{1}{n} \frac{\partial \Pi_{H=0}}{\partial T}+\hat{S}_{\mathrm{NP}}-e \xi_{0} S_{e}^{s t}+k\left(S_{1}-S_{2}\right)\right]
$$

with the parameters $S_{1}$ and $S_{2}$ given by :

$$
S_{1}=\frac{\xi_{e} L\left(\xi_{e}\right)}{1-\lambda \gamma L^{\prime}\left(\xi_{e}\right)}-\ln \left(\frac{\sinh \left(\xi_{e}\right)}{\xi_{e}}\right)
$$

and

$$
S_{2}=\beta_{\lambda} \frac{\xi_{e} L^{\prime}\left(\xi_{e}\right)}{L\left(\xi_{e}\right)}
$$

These equations, while different from the in-field equations of the diffusion coefficient, however involve the same two parameters $\lambda$ (mean field parameter, here fixed to 0.22) and $\gamma$ (dipolar interaction parameter). We thus use in Fig. 5 the same fitting parameter $\gamma$ as in Fig. 4, which gives a quite reasonable agreement with experimental results, both for positive $S_{T}(H=0)\left(\mathrm{TBuA}^{+}\right.$ counter-ions) and for negative $S_{T}(H=0)\left(\mathrm{Na}^{+}\right.$counter-ions). 


\section{Summary - Perspectives}

Thermodiffusive properties of aqueous dispersions based on citrate-coated NPs with various kinds of counter-ions are here explored. The structural charge of the NPs, which is partially compensated by some condensed counter-ions, imparts a strong interparticle electrosteric repulsion to the dispersions. Their colloidal stability is probed by small angle x-rays scattering in zero magnetic field. It allows the determination of the second virial coefficient $A_{2}$ of the osmotic pressure (related to the two-bodies term of the interparticle potential). In-field stability is probed by light scattering up to $300 \mathrm{kA} \mathrm{m}^{-1}$.

Using Forced Rayleigh Scattering, it is shown that the direction of NP's thermodiffusion in a gradient of temperature is closely connected with the nature of the counter-ions condensed on the NPs. Small hydrophilic counter-ions such as $\mathrm{Na}^{+}$lead to a thermophilic behaviour (Soret coefficient $S_{\mathrm{T}}<0$ ) while big hydrophobic ions such as $\mathrm{TBuA}^{+}$lead to a thermophobic behaviour $\left(S_{\mathrm{T}}>0\right)$. Increasing the NP's volume fraction $\Phi$ at constant ionic strength decreases the Soret coefficient in absolute value whatever the nature of counter-ions, without changing the sign of $S_{\mathrm{T}}$, thus the direction of thermodiffusion in the temperature gradient.

The volume fraction dependence of $S_{\mathrm{T}}$ is well described by the proposed model, involving a Seebeck (thermoelectric) contribution resulting from the electric field produced by all ionic species that are present in the temperature gradient. This Seebeck contribution to $S_{\mathrm{T}}$ appears particularly intricate, while its value at $\Phi=0$ is directly connected to the difference of entropy of transfer $\hat{S}_{+}-\hat{S}_{-}$between the two kinds of free ions present in the dispersion, its volume fraction dependence is, after a first decrease in absolute value, very difficult to predict.

The model indeed also involves the single-particle thermophoretic contribution of the NPs (or equivalently their Eastman entropy of transfer $\hat{S}_{\mathrm{NP}}$ ) which is here determined. It is (in absolute value) of the same order of magnitude as the thermoelectric contribution, and it is found directly proportional to the Eastman entropy of transfer of the counter-ions (here $\hat{S}_{+}$). The single-particle thermophoretic contribution $\hat{S}_{\mathrm{NP}}$ contains several ionic-shielding contributions associated to electrostatic and hydration of the NP's ionic shell. For $\mathrm{Li}^{+}, \mathrm{Na}^{+}$and $\mathrm{TMA}^{+}$counterions, the hydration contribution is found negative as expected, while it is found positive for the big $\mathrm{TBuA}^{+}$counter-ions. It would be interesting in the future to probe for these counter-ions, the hydration contribution as a function of the volume of the ionic-shell (thus as a function of the ionic strength and/or NP's diameter) as it has already been performed in ${ }^{42}$ for $\mathrm{Na}^{+}$counter-ions.

The observed in-field anisotropy of diffusion coefficient $D_{m}$ and of Soret coefficient $S_{\mathrm{T}}$ is well described by the proposed meanfield model, with only one (and the same) adjustable $\gamma$ parameter. The application of a magnetic field, as well, does not produce any modification in the sign of the Soret coefficient which keeps its zero-concentration sign whatever $\Phi$ and applied $\vec{H}$, the direction of thermodiffusion in the temperature gradient remaining ruled by the nature of the counter-ions. However whatever the system an applied field perpendicular to $\vec{\nabla} T$ always increases the Soret coefficient $S_{\mathrm{T}}$ in absolute value, suggesting an increase of the Seebeck contribution in thermoelectric measurements performed under an applied field perpendicular to the temperature gradient $\vec{\nabla} T$.

\section{Conflicts of interest}

There are no conflicts of interest to declare.

\section{Acknowledgements}

The authors deeply thank A. Anfry for the NP's electrophoretic characterizations and acknowledge $\mathrm{M}$. Bonetti for very fruitful discussions. They are grateful to ANR TE-FLIC (Grant $\mathrm{n}^{\circ}$ ANR12-PRGE-0011-01) for its financial support and in particular for the post-doc of M.K.. C.L.F. is also very grateful for CAPES Grant $\mathrm{n}^{\circ}$ 99999.001111/2014-00 and for CAIQ/UnB support. This work has been supported by European Union's Horizon 2020 research and innovation programme under the grant agreement $\mathrm{n}^{\circ} 731976$ (MAGENTA), by program CAPES-COFECUB $n^{\circ} 714 / 11$ between France and Brazil, and by PHC Osmose 2018 contract $n^{\circ} 40033 S$ between France and Latvia.

\section{References}

1 R. Rosensweig, Ferrohydrodynamics, Cambridge monographs on mechanics and applied mathematics - Cambridge University Press - Cambridge, 1985.

2 Magnetic Fluids and Applications Handbook, ed. B. Berkovski, Begell House Inc. Publ., New York, 1996.

3 E. Blums, A. Cēbers and M. Maiorov, Magnetic Liquids., W. de G. Gruyter - New York, 1997.

4 Magnetically Controllable Fluids and Their Applications, ed. S. Odenbach, Springer Verlag, Berlin, 2010.

5 B. T. Huang, M. Roger, M. Bonetti, T. J. Salez, C. WiertelGasquet, E. Dubois, R. C. Gomes, G. Demouchy, G. Mériguet, V. Peyre, M. Kouyaté, C. L. Filomeno, J. Depeyrot, F. A. Tourinho, R. Perzynski and S. Nakamae, J. Chem. Phys., 2015, 143, 054902 1-5.

6 T. J. Salez, B. T. Huang, M. Rietjens, M. Bonetti, C. WiertelGasquet, M. Roger, C. L. Filomeno, E. Dubois, R. Perzynski and S. Nakamae, Phys. Chem. Chem. Phys., 2017, 19, 94099416.

7 S. A. Putnam and D. G. Cahill, Langmuir, 2005, 21, 53175323.

8 A. Würger, Phys. Rev. Lett., 2008, 101, 108302.

9 A. Majee and A. Würger, Phys. Rev. E, 2011, 83, 061403 pp. 1-6.

10 A. Majee, PhD thesis, Université Bordeaux I, 2012.

11 M. Reichl, M. Herzog, A. Gotz and D. Braun, Phys. Rev. Lett., 2014, 112, 198101 pp.1-5.

12 K. A. Eslahian, A. Majee, M. Maskos and A. Würger, Soft Matter, 2014, 10, 1931-1936.

13 C. Goupil, W. Seifert, K. Zabrocki, E. Müller and G. Snyder, Entropy, 2011, 13, 1481-1517.

14 C. Ludwig, Sitzungsber. Akad. Wiss. Wien Math.- Naturwiss., 1856, 20, 539.

15 C. Soret, Arch. Sci. Phys. Nat. Genève, 1879, 3, 48. 
16 J. N. Agar, C. Y. Mou and J. L. Lin, J. Phys. Chem., 1989, 93, 2079-2082.

17 N. Takeyama and K. Nakashima, J. Solution Chem., 1988, 17, 305.

18 F. Römer, Z. Wang, S. Wiegand and F. Bresme, J. Phys. Chem. B, 2013, 117, 8209-8222.

19 S. di Lecce, T. Albrecht and F. Bresme, Sci. Rep., 2017, 7, 44833 1-9.

20 S. di Lecce and F. Bresme, J. Phys. Chem. B, 2018, 122, 16621668.

21 A. Sehnem, D. Niether, S. Wiegand and A. M. F. Neto, J. Phys. Chem. B, 2018, 122, 4093-4100.

22 R. Hu, B. Cola, N. Haram, J. Barisci, S. Lee, S. Stougton, G. Wallace, C. Too, M. Thomas, A. Gestos, M. dela Cruz, J. Ferraris, A. Zakhidov and R. Baughman, Nano Letters, 2010, 10, 838-846.

23 V. Zinovyeva, S. Nakamae, M. Bonetti and M. Roger, ChemElectroChem, 2014, 1, 426-430.

24 M. Dupont, D. MacFarlane and J. Pringle, Chem. Commun., 2017, 53, 6288-6302.

25 D. Al-Masri, M. Dupont, R. Yunis, D. R. MacFarlane and J. M. Pringle, Electrochim. Acta, 2018, 269, 714-723.

26 C. L. Filomeno, M. Kouyaté, V. Peyre, G. Demouchy, A. F. C. Campos, R. Perzynski, F. A. Tourinho and E. Dubois, J. Phys. Chem. C, 2017, 121, 5539-5550.

27 C. L. Filomeno, M. Kouyaté, F. Cousin, G. Demouchy, E. Dubois, L. Michot, G. Mériguet, R. Perzynski, V. Peyre, J. Sirieix-Plénet and F. A. Tourinho, J. Magn. Magn. Mat., 2017, 431, 2-7.

28 E. Blums, S. Odenbach, A. Mezulis and M. Maiorov, Physics of Fluids, 1998, 10, 2155-2163.

29 E. Blums, J. Magn. Magn. Mat., 2005, 289, 246-249.

30 L. Sprenger, A. Lange and S. Odenbach, Physics of Fluids, 2014, 26, 022001 1-16.

31 E. Dubois, R. Perzynski, F. Boué and V. Cabuil, Langmuir, 2000, 16, 5617-5625.

32 G. Mériguet, F. Cousin, E. Dubois, F. Boué, A. Cēbers, B. Farago and R. Perzynski, J. Phys. Chem. B, 2006, 110, 4378-4386.

33 G. Mériguet, E. Wandersman, E. Dubois, A. Cēbers, J. A. Gomes, G. Demouchy, J. Depeyrot, A. Robert and R. Perzynski, Magnetohydrodynamics, 2012, 48, 415-425.

34 G. Demouchy, A. Mezulis, A. Bée, D. Talbot, J.-C. Bacri and A. Bourdon, J. Phys. D: Appl. Phys., 2004, 37, 1417-1428.

35 Y. Nagasaka, T. Hatakeyama, M. Osuka and A. Nagashima, Rev. Sci. Instrum., 1988, 59, 1156.

36 Y. Marcus, J. Solution Chem., 1994, 23, 831-848.

37 Y. Marcus, J. Solution Chem., 2008, 37, 1071-1098.

38 Y. Marcus, Chem. Rev., 2009, 109, 1346-1370.

39 K. A. Eslahian and M. Maskos, Colloids Surf. A, 2012, 413, 65-70.

40 B. Huang, S. Muy, S. Feng, Y. Katayama, Y.-C. Lu, G. Chen and Y. Shao-Horn, Phys. Chem. Chem. Phys., 2018, 20, 1568015686.
41 D. Bhowmik, N. Malikova, G. Mériguet, O. Bernard, J. Teixeira and P. Turq, Phys. Chem. Chem. Phys., 2014, 16, 1344713457.

42 R. Cabreira-Gomes, A. F. da Silva, M. Kouyaté, G. Demouchy, G. Mériguet, R. Aquino, E. Dubois, S. Nakamae, M. Roger, J. Depeyrot and R. Perzynski, Phys. Chem. Chem. Phys., 2018, 20, 16402-16413.

43 J. K. G. Dhont, S. Wiegand, S. Duhr and D. Braun, Langmuir, 2007, 23, 1674-1683.

44 J. K. G. Dhont and W. J. Briels, Eur. Phys. J. E, 2008, 25, 6176.

45 T. J. Salez, S. Nakamae, R. Perzynski, G. Mériguet, A. Cēbers and M. Roger, Entropy, 2018, 20, 405 1-27.

46 J. C. Bacri, A. Cēbers, A. Bourdon, G. Demouchy, B. M. Heegaard and R. Perzynski, Phys. Rev. Lett., 1995, 74, 5032-5035.

47 J. C. Bacri, A. Cēbers, A. Bourdon, G. Demouchy, B. M. Heegaard, , B. Kashevsky and R. Perzynski, Phys. Rev. E, 1995, 52, 3936-3942.

48 R. Massart, C. R. Acad. Sci. Paris, Série C, 1980, 291, 1-3.

49 R. Massart, I.E.E.E. Trans. Mag. Magn., 1981, 17, 1247-1248.

50 J. C. Bacri, R. Perzynski, D. Salin, V. Cabuil and R. Massart, J. Magn. Magn. Mat., 1990, 85, 27-32.

51 E. Wandersman, A. Cēbers, E. Dubois, G. Mériguet, A. Robert and R. Perzynski, Soft Matter, 2013, 9, 11480-11489.

52 N. F. Carnahan and K. E. Starling, J. Chem. Physics, 1970, 53, 600.

53 J. A. Barker and D. Henderson, J. Chem. Phys., 1967, 47, 4714-4721.

54 D. Zablotsky, A. Mezulis and E. Blums, C. R. Mecanique, 2013, 341, 449-454.

55 D. Zablotsky, PhD thesis, Univ. of Latvia - Riga, 2012.

56 R. Piazza and A. Parola, J. Phys.: Condens. Matter, 2008, 20, 153102 pp. 1-18.

57 J. Burelbach, D. Frenkel, I. Pagonabarraga and E. Eiser, Eur. Phys. J. E, 2017, 41, 7 p.1-12.

58 J. K. G. Dhont, J. Chem. Phys., 2004, 120, 1632-1641.

59 J. K. G. Dhont, J. Chem. Phys., 2004, 120, 1642-1653.

60 G. Mériguet, E. Dubois, M. Jardat, A. Bourdon, G. Demouchy, V. Dupuis, B. Farago, R. Perzynski and P. Turq, J. Phys.: Cond. Matt., 2006, 18, S2685-S2696.

61 F. Gazeau, F. Boué, E. Dubois and R. Perzynski, J. Phys.: Condens. Matter, 2003, 15, S1305-S1334.

62 A. O. Ivanov and O. B. Kuznetsova, Phys. Rev. E, 2001, 64, 041405 1-12.

63 K. I. Morozov and A. V. Lebedev, J. Magn. Magn. Mat., 1990, 85, 51.

64 D. Vigolo, S. Buzzaccaro and R. Piazza, Langmuir, 2010, 26, 7792-7801.

65 M. Yang and M. Ripoll, Soft Matter, 2013, 9, 4661-4671.

66 S. Duhr and D. Braun, Proc. Natl. Acad. Sci. U.S.A., 2006, 103, 19678-19682.

67 H. Ning, J. K. G. Dhont and S. Wiegand, Langmuir, 2008, 24, 2426-2432. 
68 Y. Marcus, Ion properties, Marcel Dekker, New York, 1997.

69 D. Dong, J. Hooper and D. Bedrov, J. Phys. Chem. B, 2017, 121, 4853-4863.
70 D. Niether, S. Di Lecce, F. Bresme and S. Wiegand, Phys. Chem. Chem. Phys., 2018, 20, 1012-1020. 